\title{
Body mass index modulates the relationship of sugar-sweetened beverage intake with serum urate concentrations and gout
}

\author{
Nicola Dalbeth ${ }^{1 *}$, Amanda Phipps-Green², Meaghan E. House ${ }^{1}$, Gregory D. Gamble ${ }^{1}$, Anne Horne ,
} Lisa K. Stamp ${ }^{3}$ and Tony R. Merriman ${ }^{2}$

\begin{abstract}
Introduction: Both sugar-sweetened beverage (SSB) intake and body mass index (BMI) are associated with elevated serum urate concentrations and gout risk. The aim of this study was to determine whether the associations of SSB intake with serum urate and gout are moderated by BMI.

Method: The effects of chronic SSB intake on serum urate and gout status were analysed in a large cross-sectional population study. The effects of an acute fructose load on serum urate and fractional excretion of uric acid (FEUA) were examined over 180 minutes in a short-term intervention study. In all analyses, the responses were compared in those with BMl $<25 \mathrm{mg} / \mathrm{kg}^{2}$ (low BMI) and $\geq 25 \mathrm{mg} / \mathrm{kg}^{2}$ (high BMl).

Results: In the serum urate analysis $(n=12,870)$, chronic SSB intake was associated with increased serum urate in the high BMI group, but not in the low BMI group $\left(P_{\text {difference }}=3.6 \times 10^{-3}\right)$. In the gout analysis $(n=2578)$, chronic high SSB intake was associated with gout in the high BMI group, but not in the low BMI group $\left(P_{\text {difference }}=0.012\right.$ ). In the acute fructose loading study $(n=76)$, serum urate was increased in the high BMI group at baseline and throughout the observation period $\left(P_{B M I}\right.$ group $\left.<0.0001\right)$, but there were similar acute serum urate increases in both $\mathrm{BMI}$ groups in response to the fructose load $\left(P_{\text {interaction }}=0.99\right)$. The baseline FEUA was similar between the two BMI groups. However, following the fructose load, FEUA responses in the BMI groups differed $\left(P_{\text {interaction }}<0.0001\right)$, with increased FEUA at 120 minutes and 180 minutes in the low BMI group and reduced FEUA at 60 minutes in the high BMl group.
\end{abstract}

Conclusions: These data suggest that BMI influences serum urate and gout risk in response to chronic SSB intake, and renal tubular uric acid handling in response to an acute fructose load. In addition to many other health benefits, avoidance of SSBs may be particularly important in those with overweight/obesity to prevent hyperuricaemia and reduce gout risk.

Trials registration: Australian Clinical Trials Registry ACTRN12610001036000. Registered 24 November 2010.

\section{Introduction}

Serum urate concentrations are influenced by a number of modifiable and non-modifiable factors. Intake of sugar-sweetened beverages is a strong modifiable risk factor for hyperuricaemia and gout [1-4]. The effect of sugar-sweetened beverage intake on serum urate has been attributed to the hepatic effects of fructose on

\footnotetext{
* Correspondence: n.dalbeth@auckland.ac.nz

'Department of Medicine, Faculty of Medical and Health Sciences, University of Auckland, 85 Park Road, Grafton, Auckland 1023, New Zealand
} Full list of author information is available at the end of the article adenosine triphosphate degradation, which in turn, induces urate production [5]. Sugar may also directly interfere with urate excretion via the hexose-uric acid transporter solute carrier family 2, facilitated glucose transporter member 9 (SLC2A9) [4].

Elevated body mass index is another modifiable risk factor for hyperuricaemia and development of gout [6], with a consistent strong relationship between central adiposity and serum urate [7], which may be mediated by the effects of insulin on renal tubular uric acid reabsorption [8]. There are accumulating data that body 
mass index can modulate the influence of nonmodifiable genetic variants on serum urate $[9,10]$. The influence of body mass index may also modulate associations of modifiable factors, such as sugar-sweetened beverage intake, with serum urate and gout. The aim of this study was to determine whether the associations between sugar-sweetened beverage intake with serum urate and gout are moderated by body mass index.

\section{Methods}

We addressed the study aim in two separate analyses: first, in a large cross-sectional population study examining the effects of chronic sugar-sweetened beverage intake on serum urate and gout, and second, in a shortterm intervention study examining the effects of an acute fructose load on serum urate and fractional excretion of uric acid. In all analyses, the responses were analysed in those with body mass index $<25 \mathrm{mg} / \mathrm{kg}^{2}$ (low body mass index group) and those with body mass index $\geq 25 \mathrm{mg} / \mathrm{kg}^{2}$ (high body mass index group).

\section{Chronic sugar-sweetened beverage intake serum urate analysis}

The effect of chronic sugar-sweetened beverage intake on serum urate in separate body mass index strata was assessed by analysing 12,870 people without gout from the Atherosclerosis Risk in Communities (ARIC), Framingham Heart Study (FHS) and New Zealand (NZ) datasets. The ARIC $(n=8,436)$ and FHS $(n=3,066)$ cohorts are US longitudinal studies described by us elsewhere [11] whereas the NZ dataset is a cross-sectional sample set recruited from 2007 to 2014 [4]. The FHS Generation 3 cohort was analysed with all measures taken at examination 1 (20022005) and all ARIC measures were taken at examination 1 (1987-1989). Sugar-sweetened beverage consumption was assigned to one of three categories - those drinking no sugar-sweetened beverages (no sugar-sweetened beverage intake group), $>0$ to $<2$ sugar-sweetened beverages per day (low sugar-sweetened beverage intake group) and $\geq 2$ sugar-sweetened beverages per day (high sugar-sweetened beverage intake group). Serum urate was regressed against the three categories in body mass index $<25 \mathrm{mg} / \mathrm{kg}^{2}$, body mass index $\geq 25 \mathrm{mg} / \mathrm{kg}^{2}$ and unstratified. All analyses were adjusted by ethnicity, age, sex, fruit intake, triglycerides, kidney disease (self-reported) and hypertension, and relatedness in FHS. Significant difference in the change in serum urate per sugar-sweetened beverage category between body mass index groups was assessed by calculating a Cochran's $Q$ test statistic and the corresponding $P$ value $\left(P_{\mathrm{Q}}\right)$. Data were analysed using STATA version 13.1 (StataCorp, College Station, TX, USA) and R 3.0.2 in the RStudio GUI version 0.98.1087 (R Foundation for Statistical Computing, Vienna, Austria).

\section{Chronic sugar-sweetened beverage intake gout analysis}

The effect of chronic sugar-sweetened beverage intake on gout status in separate body mass index strata was assessed by analysing 2,578 people $(\mathrm{n}=1,368$ without gout and 1,210 with gout) from the NZ dataset [4]. Gout was clinically ascertained using the 1977 American Rheumatism Association preliminary gout classification criteria [12]. A logistic regression of sugarsweetened beverage intake (based on the three categories: $0,>0$ to $<2,>2$ ) with gout status was performed to determine the odds ratio for gout. All analyses were adjusted by ethnicity, age, sex, fruit intake, triglycerides, kidney disease and hypertension. Significant difference in the odds ratios for gout per sugar-sweetened beverage category between body mass index groups was assessed by calculating a Cochran's $Q$ test statistic and the corresponding $P$ value $\left(P_{\mathrm{Q}}\right)$. Data were analysed using STATA version 13.1 and R 3.0.2 in the RStudio GUI version 0.98.1087.

\section{Acute fructose intake analysis}

The methods of the acute fructose intake study have been previously reported [13]. Exclusion criteria were: gout, diabetes mellitus or fructose intolerance, diuretic use, fasting glucose $>6 \mathrm{mmol} / \mathrm{L}$. Following an overnight fast, 76 healthy volunteers consumed a sugar solution between 8 a.m. and 9 a.m., and blood was obtained prior to ingestion and then 30 minutes, 60 minutes, $120 \mathrm{mi}-$ nutes, and 180 minutes after ingestion. Urine was obtained at each time point for testing of urate and creatinine to allow calculation of fractional excretion of uric acid (uric acid clearance/creatinine clearance expressed as a percentage). Weight and height were measured at the start of the study visit, and body mass index was calculated. The sugar solution of $300 \mathrm{kcal} / 300 \mathrm{ml}$ was consumed within 10 minutes, according to the protocol of Akhavan and Anderson for fructose-induced hyperuricaemia [14]. This solution contained $80 \%$ fructose and $20 \%$ glucose (64 g fructose and $16 \mathrm{~g}$ glucose). The study was approved by the New Zealand Multiregional Ethics Committee, and each participant gave written informed consent.

Data were analysed using a mixed models approach to repeated measures. Significant main and interaction effects were explored using the method of Tukey. Sex, ethnicity and triglycerides were adjusted for within the models. For change in serum urate and other biochemical variables, a mixed models analysis of covariance (ANCOVA) approach to repeated measures was used. For ANCOVA, the dependent variable was change from baseline, and baseline level was included as a covariate. Analyses were performed using SAS v 9.2 (SAS Institute Inc., Cary, NC, USA). $P<0.05$ was considered significant and all tests were two-tailed. 


\section{Results}

\section{Chronic sugar-sweetened beverage intake and serum urate}

The characteristics of participants in the chronic sugarsweetened beverage intake and serum urate analysis are shown in Table 1 . The majority of participants were of Caucasian ethnicity $(n=10,443)$, with smaller numbers of participants of New Zealand Polynesian $(n=918)$ and African American $(n=1,509)$ ethnicity. More than half (61.1\%) of participants had a body mass index $\geq 25 \mathrm{~kg} / \mathrm{m}^{2}$. The mean sugar-sweetened beverage intake in the low body mass index group was 1.1 drinks/day and in the high body mass index group was 1.2 drinks/day. Serum urate was higher in the high body mass index group compared to the low body mass index group.

In this analysis, high sugar-sweetened beverage intake was associated with higher serum urate in the entire group (compared with no sugar-sweetened beverage intake, body mass index-adjusted $P=0.11$ for low sugarsweetened beverage intake and $P=1.9 \times 10^{-3}$ for high sugar-sweetened beverage intake, Table 2). There was no association between sugar-sweetened beverage intake and serum urate in the low body mass index group $(P=0.20$ for low sugar-sweetened beverage intake and $P=0.67$ for high sugar-sweetened beverage intake). In contrast, increased sugar-sweetened beverage intake was associated with higher serum urate in the high body mass index group $\left(P=9.4 \times 10^{-4}\right.$ for low sugarsweetened beverage intake and $P=7.2 \times 10^{-6}$ for high sugar-sweetened beverage intake). The difference in serum urate according the sugar-sweetened beverage categories was significantly greater in the high body mass index group than in the low body mass index group $\left(P_{\text {difference }}=3.6 \times 10^{-3}\right)$. The difference in serum urate was higher in the high body mass index group for both low sugar-sweetened beverage intake $\left(P_{\text {difference }}=\right.$ $\left.1.4 \times 10^{-3}\right)$ and high sugar-sweetened beverage intake $\left(P_{\text {difference }}=9.3 \times 10^{-4}\right)$.

\section{Chronic sugar-sweetened beverage intake and gout status}

The characteristics of participants in the chronic sugarsweetened beverage intake and gout analysis are shown in Table 3. The majority of participants with gout (76.8 \%) were on urate-lowering therapy. There were differences between cases and controls with respect to sex, age, ethnicity, serum urate, triglycerides, body mass index, and history of hypertension and kidney disease (Table 3).

In the low body mass index group, after adjustment for all these variables, low sugar-sweetened beverage intake was associated with a reduced odds ratio (OR) for gout (OR 0.43, $P=0.043$ ) compared to no intake, and there was no increase in the odds ratio for high sugarsweetened beverage intake (OR 0.64, $P=0.31$ ) (Table 4). In contrast, high sugar-sweetened beverage intake was associated with a higher odds ratio for gout (OR 1.33, $P=0.033)$ in the high body mass index group. The

Table 1 Clinical features of all participants in the chronic sugar-sweetened beverage intake urate analysis

\begin{tabular}{|c|c|c|c|c|c|}
\hline & Percentage with data & $\begin{array}{l}\text { All } \\
(\mathrm{n}=12,870)\end{array}$ & $\begin{array}{l}\mathrm{BMI}<25 \\
(\mathrm{n}=5,002)\end{array}$ & $\begin{array}{l}\mathrm{BMI} \geq 25 \\
(\mathrm{n}=7,862)\end{array}$ & $P$ \\
\hline Age, years & 100 & $49.5(10.1)$ & $48.9(10.4)$ & $49.9(9.9)$ & $9 \times 10^{-9^{*}}$ \\
\hline Male sex, $\mathrm{n}(\%)$ & 100 & $6030(46.9)$ & $1740(34.8)$ & $4288(54.5)$ & $<1.0 \times 10^{-5^{* *}}$ \\
\hline NZ East Polynesian dataset, $\mathrm{n}(\%)$ & 100 & $583(4.5)$ & $96(1.9)$ & $487(6.2)$ & $<1.0 \times 10^{-5^{* *}}$ \\
\hline NZ West Polynesian dataset, n (\%) & 100 & $271(2.1)$ & $16(0.3)$ & $255(3.2)$ & \\
\hline NZ Mixed East/West Polynesian dataset, n (\%) & 100 & $64(0.5)$ & $4(0.1)$ & $60(0.8)$ & \\
\hline NZ Caucasian dataset, n (\%) & 100 & $450(3.5)$ & $152(3.0)$ & $298(3.8)$ & \\
\hline ARIC Caucasian dataset, n (\%) & 100 & $6927(53.8)$ & $2930(58.6)$ & $3996(50.8)$ & \\
\hline FHS Caucasian dataset, n (\%) & 100 & $3066(23.8)$ & $1380(27.6)$ & $1686(21.4)$ & \\
\hline ARIC African American dataset, n (\%) & 100 & $1509(11.7)$ & $424(8.5)$ & $1080(13.7)$ & \\
\hline Serum urate, $\mathrm{mmol} / \mathrm{L}$ & 98.4 & $0.33(0.09)$ & $0.30(0.07)$ & $0.36(0.08)$ & $<1.0 \times 10^{-300}$ \\
\hline Triglycerides, mmol/L & 98.0 & $1.79(1.52)$ & $1.45(1.04)$ & $2.01(1.72)$ & $<1.0 \times 10^{-300}$ \\
\hline Body mass index, $\mathrm{kg} / \mathrm{m}^{2}$ & 99.9 & $27.1(5.4)$ & $22.4(1.8)$ & $30.1(4.8)$ & $<1.0 \times 10^{-300}$ \\
\hline Fruit intake, pieces/day & 99.8 & $1.47(1.31)$ & $1.45(1.32)$ & $1.48(1.31)$ & $0.32^{*}$ \\
\hline Kidney disease, n (\%) & 96.3 & $134(1.1)$ & $34(0.7)$ & $100(1.3)$ & $1.4 \times 10^{-3^{* *}}$ \\
\hline Hypertension, n (\%) & 99.3 & $1541(12.1)$ & $395(7.9)$ & $1143(14.7)$ & $<1.0 \times 10^{-5^{* *}}$ \\
\hline
\end{tabular}

Unless specified, data are presented as mean (SD)

$B M I$ body mass index, NZ New Zealand, ARIC Atherosclerosis Risk in Communities, FHS Framingham Heart Study

"Two-sample $t$ test with unequal variances

${ }^{* *}$ Chi-square test 
Table 2 Difference in serum urate concentration for chronic sugar-sweetened beverage intake stratified by body mass index (BMI) group

\begin{tabular}{|c|c|c|c|c|c|c|}
\hline & \multirow[b]{2}{*}{ SSB drinks/day } & \multicolumn{2}{|l|}{ Per SSB category } & \multicolumn{3}{|l|}{ Overall SSB category } \\
\hline & & $\begin{array}{l}\Delta \text { in serum urate, } \\
95 \% \mathrm{Cl}(\mathrm{mmol} / \mathrm{L})\end{array}$ & $P^{* *}$ & $\begin{array}{l}\Delta \text { in serum urate, } \\
95 \% \mathrm{Cl}(\mathrm{mmol} / \mathrm{L})\end{array}$ & $P$ & $\begin{array}{l}P_{\text {difference }} \text { between } \\
\mathrm{BMl}<25 \text { and } \geq 25\end{array}$ \\
\hline \multirow[t]{3}{*}{ All participants $(n=12,081)^{*}$} & 0 & - & - & $0.004(0.002-0.006)$ & $9.7 \times 10^{-4}$ & - \\
\hline & $>0$ to $<2$ & $0.004(0.000-0.008)$ & 0.11 & & & \\
\hline & $\geq 2$ & $0.009(0.005-0.014)$ & $1.9 \times 10^{-3}$ & & & \\
\hline \multirow[t]{3}{*}{$\mathrm{BMI}<25(\mathrm{n}=4,731)$} & 0 & - & - & $-0.000(-0.004-0.003)$ & 0.97 & $3.6 \times 10^{-3}$ \\
\hline & $>0$ to $<2$ & $-0.004(-0.010-0.002)^{\dagger}$ & 0.20 & & & \\
\hline & $\geq 2$ & $-0.002(-0.009-0.006)^{\dagger+}$ & 0.67 & & & \\
\hline \multirow[t]{3}{*}{$\mathrm{BMI} \geq 25(\mathrm{n}=7,350)$} & 0 & - & - & $0.007(0.004-0.010)$ & $1.4 \times 10^{-5}$ & \\
\hline & $>0$ to $<2$ & $0.010(0.004-0.015)^{\dagger}$ & $9.4 \times 10^{-4}$ & & & \\
\hline & $\geq 2$ & $0.015(0.008-0.021)^{t \dagger}$ & $7.2 \times 10^{-6}$ & & & \\
\hline
\end{tabular}

The difference in serum urate in the overall sugar-sweetened beverage (SSB) category is the average difference from sugar-sweetened beverage category 1 to category 2 to category 3 (i.e. 0 , to $>0$ to $<2$, to $\geq 2$ ). All analysis adjusted by sample set (Atherosclerosis Risk in Communities (ARIC) Caucasian, Framingham Heart Study (FHS) Caucasian, New Zealand (NZ) Caucasian, East Polynesian, West Polynesian, Mixed East Polynesian/West Polynesian, ARIC African American), age, sex, fruit intake (continuous variable), kidney disease, hypertension, triglycerides, and relatedness

*Analysis adjusted by BMI

${ }^{* *}$ Compared with referent group (0 SSB/day)

${ }^{\dagger} P_{\text {difference }}$ between BMI groups $=1.4 \times 10^{-3}$

${ }^{\dagger+} P_{\text {difference }}$ between BMI groups $=9.3 \times 10^{-4}$

odds ratios according to the sugar-sweetened beverage categories were significantly greater in the high body mass index group than in the low body mass index group $\left(P_{\text {difference }}=0.012\right)$. The difference in the odds ratios for gout were significantly different between the body mass index groups for both low sugar-sweetened beverage intake $\left(P_{\text {difference }}=5.9 \times 10^{-4}\right)$ and high sugar-sweetened beverage intake $\left(P_{\text {difference }}=0.028\right)$.

\section{Acute fructose intake}

The characteristics of participants in the acute fructose intake analysis are shown in Table 5 . As previously reported, the study participants were of European $(\mathrm{n}=25)$, Māori $(\mathrm{n}=25)$ and Pacific $(\mathrm{n}=26)$ ethnicity. More than half (59\%) of participants had a body mass index $\geq 25 \mathrm{~kg} / \mathrm{m}^{2}$. There were fewer European and more Pacific participants in the high body mass index group. All analyses were adjusted for sex and ethnicity.

In the acute fructose feeding study, serum urate was higher in the high body mass index group at baseline and throughout the observation period

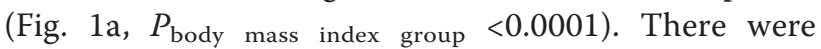
similar acute serum urate increases in both body mass index groups in response to the fructose load

Table 3 Clinical features of participants in the gout analysis

\begin{tabular}{|c|c|c|c|c|c|}
\hline & Percentage with data & $\begin{array}{l}\text { All } \\
(n=2,578)\end{array}$ & $\begin{array}{l}\text { Controls } \\
(1,368)\end{array}$ & $\begin{array}{l}\text { Cases } \\
(1,210) \\
\end{array}$ & $P$ \\
\hline Age, years & 100 & $52.1(16.4)$ & $47.0(16.5)$ & $57.8(14.4)$ & $4.9 \times 10^{-66^{*}}$ \\
\hline Male sex, n (\%) & 100 & $1739(67.5)$ & $740(54.1)$ & 999 (82.6) & $<1.0 \times 10^{-5^{* *}}$ \\
\hline NZ East Polynesian dataset, n (\%) & 100 & $901(35.0)$ & $583(42.6)$ & $319(26.4)$ & $<1.0 \times 10^{-5^{* *}}$ \\
\hline NZ West Polynesian dataset, n (\%) & 100 & $597(23.2)$ & $271(19.8)$ & $326(26.9)$ & \\
\hline NZ Mixed East/West Polynesian dataset, n (\%) & 100 & $92(3.6)$ & $64(4.7)$ & $28(2.3)$ & \\
\hline NZ Caucasian dataset, n (\%) & 100 & $987(38.3)$ & $450(32.9)$ & $537(44.4)$ & \\
\hline Serum urate at the time of recruitment, $\mathrm{mmol} / \mathrm{L}$ & 86.5 & $0.39(0.11)$ & $0.37(0.10)$ & $0.43(0.11)$ & $5.9 \times 10^{-37^{*}}$ \\
\hline Triglycerides, $\mathrm{mmol} / \mathrm{L}$ & 86.9 & $2.14(1.4)$ & $1.93(1.18)$ & $2.39(1.60)$ & $2.3 \times 10^{-14^{*}}$ \\
\hline Body mass index, $\mathrm{kg} / \mathrm{m}^{2}$ & 100 & $32.4(7.6)$ & $31.3(7.5)$ & $33.6(7.6)$ & $2.6 \times 10^{-15^{*}}$ \\
\hline Fruit intake, pieces/day & 98.9 & $2.9(4.6)$ & $2.8(4.2)$ & $3.0(5.0)$ & $0.27^{*}$ \\
\hline Kidney disease, n (\%) & 98.0 & $295(11.7)$ & $57(4.3)$ & $238(20.1)$ & $<1.0 \times 10^{-5^{* *}}$ \\
\hline Hypertension, n (\%) & 98.3 & $975(38.5)$ & $309(23.0)$ & $666(55.9)$ & $<1.0 \times 10^{-5^{* *}}$ \\
\hline
\end{tabular}

Unless specified, data are presented as mean (SD)

NZ New Zealand

*Two-sample $t$ test with unequal variances

${ }^{* *}$ Chi-square test 
Table 4 Difference in risk of gout for chronic sugar-sweetened beverage intake stratified by body mass index (BMI) group

\begin{tabular}{|c|c|c|c|c|c|c|}
\hline & \multirow[b]{2}{*}{ SSB drinks/day } & \multicolumn{2}{|l|}{ Per SSB category } & \multicolumn{3}{|l|}{ Overall SSB category } \\
\hline & & Odds ratio $(95 \% \mathrm{Cl})$ & $P^{* *}$ & Odds ratio $(95 \% \mathrm{Cl})$ & $P$ & $P_{\text {difference }}$ between $\mathrm{BMI}<25$ and $\geq 25$ \\
\hline \multirow[t]{3}{*}{ All participants $(n=2,144)^{*}$} & 0 & - & - & $1.09(0.96-1.23)$ & 0.19 & - \\
\hline & $>0$ to $<2$ & $0.83(0.64-1.07)$ & 0.15 & & & \\
\hline & $\geq 2$ & $1.20(0.93-1.54)$ & 0.16 & & & \\
\hline \multirow[t]{3}{*}{$\mathrm{BMI}<25(\mathrm{n}=304)$} & 0 & - & - & $0.74(0.49-1.13)$ & 0.16 & 0.012 \\
\hline & $>0$ to $<2$ & $0.43(0.19-0.97)^{\dagger}$ & 0.043 & & & \\
\hline & $\geq 2$ & $0.64(0.28-1.50)^{+\dagger}$ & 0.31 & & & \\
\hline \multirow[t]{3}{*}{$\mathrm{BMI} \geq 25(\mathrm{n}=1,840)$} & 0 & - & - & $1.15(1.01-1.32)$ & 0.035 & \\
\hline & $>0$ to $<2$ & $0.92(0.70-1.21)^{\dagger}$ & 0.55 & & & \\
\hline & $\geq 2$ & $1.33(1.02-1.74)^{\dagger \dagger}$ & 0.033 & & & \\
\hline
\end{tabular}

The difference in gout risk in the overall sugar-sweetened beverage (SSB) category is the average difference from sugar-sweetened beverage category 1 to category 2 to category 3 (i.e. 0 , to $>0$ to $<2$, to $\geq 2$ ). All analysis adjusted by age, sex, fruit intake (continuous variable), kidney disease, hypertension, triglycerides, and ethnicity (New Zealand (NZ) Caucasian, East Polynesian, West Polynesian and Mixed East/West Polynesian)

*Analysis adjusted by BMI

${ }^{* *}$ Compared with referent group (0 SSB/day)

${ }^{\dagger} P_{\text {difference }}$ between BMI groups $=5.9 \times 10^{-4}$

${ }^{+{ }^{+}} P_{\text {difference }}$ between BMI groups $=0.028$

(Fig. $1 \mathrm{~b}, P_{\text {interaction }}=0.99$ ). The fractional excretion of uric acid was similar between the two body mass index groups at baseline (Fig. 1c, Tukey-Kramer adjusted $P=1.0)$. The fractional excretion of uric acid in the low body mass index group increased significantly from baseline at 120 minutes and 180 minutes after the fructose load (Fig. 1c and d, Tukey-Kramer adjusted $P=0.003$ and $<0.0001$ respectively). In contrast, the fractional excretion of uric acid in the high body mass index group decreased significantly from baseline at 60 minutes after the fructose load (Fig. 1c and d, Tukey-Kramer adjusted $P=0.0002$ ), and no significant increase in fractional excretion of uric acid from baseline was observed even after 180 minutes of fructose intake. The fractional excretion of uric acid responses in the body mass index groups differed significantly following the fructose load (Fig. 1c and d, $P_{\text {interaction }}<0.0001$ ).

\section{Discussion}

This study has shown that body mass index influences urate homeostasis associated with sugar-sweetened beverage intake. With low body mass index, chronic sugarsweetened beverage intake is associated with negligible difference in serum urate. This may be due to the ability of the renal tubule to rapidly increase uric acid excretion following an event that increases serum urate, thus normalising serum concentrations. In contrast, for those with high body mass index, chronic sugar-sweetened

Table 5 Clinical features of participants in the acute fructose loading study

\begin{tabular}{|c|c|c|c|c|}
\hline & All $(n=76)$ & $\mathrm{BMI}<25(\mathrm{n}=31)$ & $\mathrm{BMI} \geq 25(\mathrm{n}=45)$ & $P$ \\
\hline Age, years & $30(17)$ & $28(14)$ & $33(19)$ & 0.22 \\
\hline Male sex, n (\%) & $45(59 \%)$ & $15(48 \%)$ & $30(67 \%)$ & 0.18 \\
\hline European ethnicity, n (\%) & $25(33 \%)$ & $16(52 \%)$ & $9(20 \%)$ & 0.009 \\
\hline Māori ethnicity, n (\%) & $25(33 \%)$ & $11(35 \%)$ & $14(31 \%)$ & 0.88 \\
\hline Pacific ethnicity, n (\%) & $26(34 \%)$ & $4(13 \%)$ & $22(49 \%)$ & 0.002 \\
\hline Serum urate, $\mathrm{mmol} / \mathrm{l}$ & $0.36(0.11)$ & $0.31(0.09)$ & $0.40(0.11)$ & 0.0003 \\
\hline Triglycerides, mmol/L & $1.24(0.78)$ & $0.93(0.34)$ & $1.44(0.92)$ & 0.0013 \\
\hline Fractional excretion of uric acid, \% & $5.82(2.15)$ & $5.97(2.46)$ & $5.70(1.97)$ & 0.60 \\
\hline Body mass index, $\mathrm{kg} / \mathrm{m}^{2}$ & $27.4(5.6)$ & $22.6(1.66)$ & $30.8(4.9)$ & $<0.0001$ \\
\hline Kidney disease, n (\%) & $1(1.3 \%)$ & $1(3.2 \%)$ & $0(0 \%)$ & 0.82 \\
\hline Hypertension, n (\%) & $6(7.9 \%)$ & $2(6.5 \%)$ & $4(8.9 \%)$ & 0.99 \\
\hline
\end{tabular}

Unless specified, data are presented as mean (SD) $B M I$ body mass index 
A

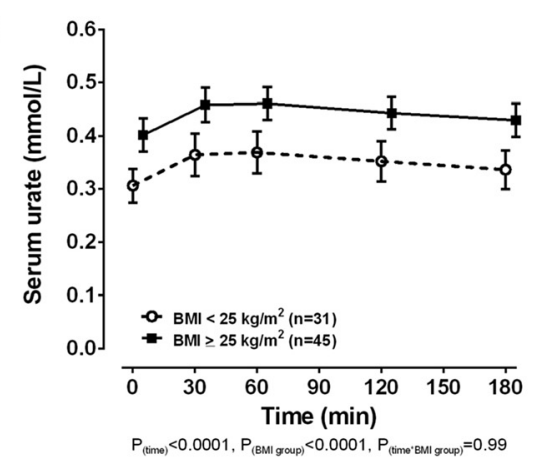

C

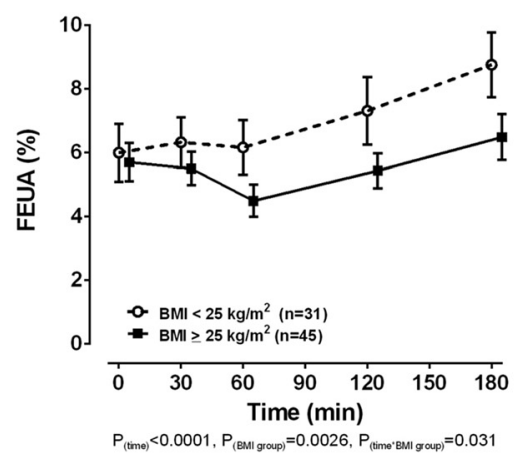

B

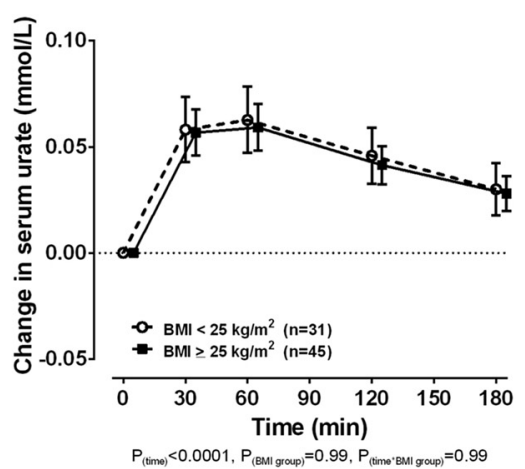

D

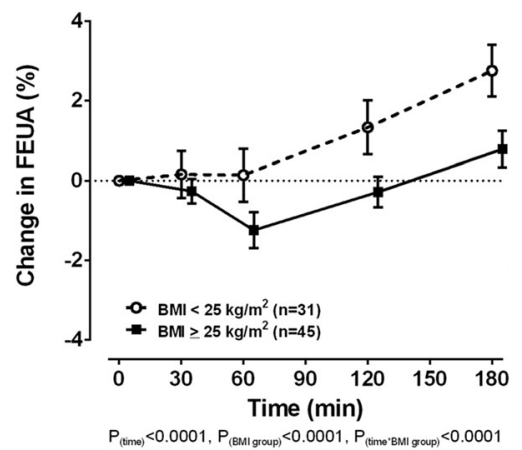

Fig. 1 The effect of body mass index (BMI) group on serum urate and fractional excretion of uric acid (FEUA) during an acute fructose load. a Serum urate concentration. b Change in serum urate concentration. c FEUA. d Change in FEUA. Data are presented as mean (95\% CI). Sex, ethnicity and triglyceride-adjusted analysis of covariance (ANCOVA) $P$ values are shown throughout

beverage intake is associated with large increases in serum urate. With high body mass index, the renal tubular urate handling response to the acute fructose is altered, with reduction in fractional excretion of uric acid and slow renal clearance of uric acid. In the context of chronic intake of dietary sugar, these renal responses may lead to prolonged serum urate elevations.

Consistent with the serum urate results, we observed that high sugar-sweetened beverage intake was associated with a higher odds ratio for gout in those with high body mass index, but not in normal weight individuals. Due to the high use of urate-lowering therapy in participants with gout, we were unable to determine whether people with gout and high body mass index have an amplified serum urate response to sugar-sweetened beverage intake, compared to normal weight people with gout. However, our gout analysis does suggest that the observed serum urate interaction between chronic sugar-sweetened beverage intake and BMI observed in the general population is clinically relevant.

In contrast to the in-hospital study by Yamashita et al. reporting lower fractional excretion of uric acid (FEUA) in people with obesity compared to normal weight control participants [15], baseline FEUA were similar between BMI groups in our acute feeding study, which studied community-dwelling healthy volunteers. However, acute FEUA responses were substantially different following the fructose load. Although higher serum urate was observed in the high body mass index group throughout the acute feeding study, we did not observe differences between body mass index groups in change in serum urate following a fructose load. This is perhaps surprising given the chronic sugar-sweetened beverage intake study and the acute fructose study fractional excretion of uric acid results. These data suggest that the hepatic urate production response to an acute fructose load is not influenced by body mass index. In the context of chronic sugarsweetened beverage intake, the ability of the renal tubule to clear urate following hepatic synthesis may account for the differential serum urate responses observed between the chronic and acute study. Unfortunately, there are currently no large publicly available databases with fractional excretion of uric acid data to further clarify whether the renal responses to chronic fructose intake differ between different body mass index groups. Differential serum urate responses to acute and chronic sugar-sweetened beverage intake have been reported previously in relation to genetic risk factors such as $S L C 2 A 9$ [4, 13], and it is possible that chronic sugar-sweetened beverage exposure influences other pathways that regulate serum urate. 


\section{Conclusions}

Consistent with recent reports showing that body mass index influences non-modifiable genetic associations with serum urate $[9,10]$, this study shows that chronic sugar-sweetened beverage intake is associated with elevated serum urate and gout status in those with high body mass index, with minimal effect observed in lean individuals. In addition to many other health benefits, avoidance of sugar-sweetened beverages may be particularly important in those with high body mass index to prevent hyperuricaemia and reduce gout risk.

\section{Abbreviations}

ANCOVA: analysis of covariance; ARIC: Atherosclerosis Risk in Communities; BMl: body mass index; FEUA: fractional excretion of uric acid; FHS: Framingham Heart Study; NZ: New Zealand; OR: odds ratio; SLC2A9: transporter solute carrier family 2, facilitated glucose transporter member 9; SSB: sugar-sweetened beverage; US: United States.

\section{Competing interests}

The authors declare that they have no competing interests.

\section{Authors' contributions}

ND (the guarantor) accepts full responsibility for the work and the conduct of the study, had access to the data, and controlled the decision to publish. ND conceived of the study, contributed to the data interpretation, and drafted the manuscript. MEH and $\mathrm{AH}$ recruited participants, coordinated study visits, and helped to revise the manuscript. MEH also managed clinical data entry. AP-G and GDG analysed the data, contributed to the data interpretation, and helped to revise the manuscript. LKS and TM conceived of the study, contributed to the data interpretation, and drafted the manuscript. All authors read and approved the final manuscript.

\section{Funding}

This study was funded by the Health Research Council of New Zealand $(11 / 1075)$.

\section{Author details}

'Department of Medicine, Faculty of Medical and Health Sciences, University of Auckland, 85 Park Road, Grafton, Auckland 1023, New Zealand. ${ }^{2}$ Department of Biochemistry, Division of Health Sciences, University of Otago, 710 Cumberland Street, Dunedin 9016, New Zealand. ${ }^{3}$ Department of Medicine, Division of Health Sciences, University of Otago, 2 Riccarton Avenue, Christchurch 8011, New Zealand.

Received: 1 May 2015 Accepted: 8 September 2015

Published online: 22 September 2015

\section{References}

1. Choi JW, Ford ES, Gao X, Choi HK. Sugar-sweetened soft drinks, diet soft drinks, and serum uric acid level: the Third National Health and Nutrition Examination Survey. Arthritis Rheum. 2008;59:109-16.

2. Choi HK, Curhan G. Soft drinks, fructose consumption, and the risk of gout in men: prospective cohort study. BMJ. 2008;336:309-12.

3. Choi HK, Willett W, Curhan G. Fructose-rich beverages and risk of gout in women. JAMA. 2010;304:2270-8.

4. Batt C, Phipps-Green AJ, Black MA, Cadzow M, Merriman ME, Topless R, et al. Sugar-sweetened beverage consumption: a risk factor for prevalent gout with SLC2A9 genotype-specific effects on serum urate and risk of gout. Ann Rheum Dis. 2014;73:2101-6.

5. Bode C, Schumacher H, Goebell H, Zelder O, Pelzel H. Fructose induced depletion of liver adenine nucleotides in man. Horm Metab Res. 1971;3:289-90.

6. Choi HK, Atkinson K, Karlson EW, Curhan G. Obesity, weight change, hypertension, diuretic use, and risk of gout in men: the health professionals follow-up study. Arch Intern Med. 2005;165:742-8.

7. Kim TH, Lee SS, Yoo JH, Kim SR, Yoo SJ, Song HC, et al. The relationship between the regional abdominal adipose tissue distribution and the serum uric acid levels in people with type 2 diabetes mellitus. Diabetol Metab Syndr. 2012;4:3.

8. Facchini F, Chen YD, Hollenbeck CB, Reaven GM. Relationship between resistance to insulin-mediated glucose uptake, urinary uric acid clearance, and plasma uric acid concentration. JAMA. 1991;266:3008-11.

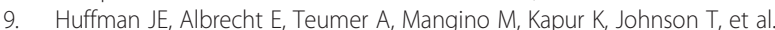
Modulation of genetic associations with serum urate levels by body-massindex in humans. PLoS One. 2015;10:e0119752.

10. Dalbeth N, House ME, Gamble GD, Pool B, Horne A, Purvis L, et al. Influence of the $A B C G 2$ gout risk $141 \mathrm{~K}$ allele on urate metabolism during a fructose challenge. Arthritis Res Ther. 2014;16:R34.

11. Rasheed H, Hughes K, Flynn TJ, Merriman TR. Mendelian randomization provides no evidence for a causal role of serum urate in increasing serum triglyceride levels. Circ Cardiovasc Genet. 2014;7:830-7.

12. Wallace SL, Robinson H, Masi AT, Decker JL, McCarty DJ, Yu TF. Preliminary criteria for the classification of the acute arthritis of primary gout. Arthritis Rheum. 1977;20:895-900.

13. Dalbeth N, House ME, Gamble GD, Horne A, Pool B, Purvis L, et al. Populationspecific influence of SLC2A9 genotype on the acute hyperuricaemic response to a fructose load. Ann Rheum Dis. 2013;72:1868-73.

14. Akhavan T, Anderson GH. Effects of glucose-to-fructose ratios in solutions on subjective satiety, food intake, and satiety hormones in young men. Am J Clin Nutr. 2007:86:1354-63.

15. Yamashita S, Matsuzawa Y, Tokunaga K, Fujioka S, Tarui S. Studies on the impaired metabolism of uric acid in obese subjects: marked reduction of renal urate excretion and its improvement by a low-calorie diet. Int J Obes. 1986;10:255-64.

\section{Submit your next manuscript to BioMed Central and take full advantage of:}

- Convenient online submission

- Thorough peer review

- No space constraints or color figure charges

- Immediate publication on acceptance

- Inclusion in PubMed, CAS, Scopus and Google Scholar

- Research which is freely available for redistribution 